\title{
Relationship Between the Eosinophil/Monocyte Ratio and Prognosis in Decompensated Heart Failure: A Retrospective Study
}

\author{
Xiehui Chen' \\ Weichao Huang ${ }^{2}$ \\ Lingyue Zhao ${ }^{3}$ \\ Yichong $\mathrm{Li}^{2}$ \\ Lili Wang ${ }^{2}$ \\ Fanrui Mo ${ }^{4}$ \\ Wenqin Guo iD ${ }^{2}$ \\ 'Department of Cardiology, Shenzhen \\ Longhua District Central Hospital, \\ Shenzhen, People's Republic of China; \\ ${ }^{2}$ Department of Cardiology, Fuwai \\ Hospital Chinese Academy of Medical \\ Sciences, Shenzhen, People's Republic of \\ China; ${ }^{3}$ Department of Ambulatory \\ Surgery, Huazhong University of Science \\ and Technology Union Shenzhen \\ Hospital, Shenzhen, People's Republic of \\ China; ${ }^{4}$ Department of Cardiology, The \\ Fourth Affiliated Hospital of Guangxi \\ Medical University, Liuzhou, People's \\ Republic of China
}

Purpose: The aim of this study was to assess the value of the eosinophil/monocyte ratio (EMR) for predicting the prognosis of decompensated heart failure (HF).

Patients and Methods: This was a retrospective cohort study. We included adults $(\geq 18$ years old) diagnosed with decompensated HF for whom EMR data were available. The patients were divided into three groups according to EMR tertiles (T1 [EMR $\leq 0.15$ ], T2 $[0.15<\mathrm{EMR} \leq 0.32]$, and $\mathrm{T} 3$ [EMR $>0.32]$ ). The primary endpoint was the composite outcome of cardiovascular death or HF rehospitalization.

Results: Initially, the records of 2264 patients with decompensated HF were screened; 1883 of these patients had EMR data and were therefore included in the study. There were 627 patients in the T1 group, 628 in the T2 group, and 628 in the T3 group. The risk of cardiovascular death or HF rehospitalization was significantly different among the three groups (Log rank test, $\mathrm{P}=0.007$ ). Compared with the $\mathrm{T} 3$ group, both the T1 group (hazard ratio $[\mathrm{HR}]: 1.50,95 \%$ confidence interval $[\mathrm{CI}]: 1.16-1.94, \mathrm{P}=0.002)$ and the T2 group (HR: $1.34,95 \% \mathrm{CI}: 1.03-1.74, \mathrm{P}=0.030$ ) had significantly higher rates of cardiovascular death or HF rehospitalization. A Cochran-Armitage test for trend showed a positive correlation between the EMR and the composite outcome of cardiovascular death or HF. There was a significant difference between the three groups in terms of cardiovascular death (Log rank test, $\mathrm{P}<0.001$ ) and HF rehospitalization (Log rank test, $\mathrm{P}=0.03$ ).

Conclusion: The EMR is positively correlated with the risk of cardiovascular death or HF rehospitalization in patients with decompensated HF. Specifically, the lower the EMR, the higher the risk of cardiovascular death or HF rehospitalization.

Keywords: decompensated heart failure, eosinophil/monocyte ratio, prognosis, cardiovascular death, heart failure rehospitalization

\section{Introduction}

Decompensated heart failure (HF) is a type of heart disease in which the ejection fraction cannot meet the body's needs due to cardiac dysfunction. The prognosis of patients with HF is poor, ${ }^{1}$ as the annual associated mortality rate for these patients is approximately $7.1 \%$ (the rate is roughly $10 \%$ during the first 3 years, but it drops to approximately $5 \%$ in subsequent years). ${ }^{2}$ Indeed, HF is the primary reason for the hospitalization of people over 65 years of age. ${ }^{3}$

Multiple studies have shown elevated inflammation markers in patients with HF. This suggests that inflammation is involved in the development and progression of $\mathrm{HF}$, and the severity of the inflammation affects a patient's prognosis. ${ }^{4,5}$ 
Eosinophils, which are produced in the bone marrow, migrate to specific organs that are involved in antibacterial immunity. Circulating eosinophils contain many cytoplasmic particles that are effective mediators of antibacterial immunity, but they are extremely toxic to heart cells; thus, these particles participate in the cardiac decompensation process. ${ }^{6}$ The eosinophil/monocyte ratio (EMR) is a new type of inflammatory marker, ${ }^{7,8}$ and it can be used to predict the prognosis of cardiovascular (CV) disease with associated underlying inflammation. For example, evidence suggests that the EMR can predict the incidence of major CV events in patients with acute coronary syndrome. ${ }^{9}$ Moreover, the EMR is associated with the prognosis and functional outcomes of patients with ischemic stroke. ${ }^{7,8}$ However, the value of the EMR for predicting the prognosis of decompensated $\mathrm{HF}$ is unknown. Therefore, the aim of this study was to evaluate the relationship between the EMR and the prognosis of decompensated $\mathrm{HF}$ using a retrospective analysis. We hypothesized that the EMR is related to the prognosis of patients with decompensated HF.

\section{Patients and Methods}

\section{Study Population}

This was a single-center retrospective cohort study. The participants were patients who were diagnosed with decompensated HF at the Fuwai Hospital Chinese Academy of Medical Sciences (Shenzhen Sun Yat-sen Cardiovascular Hospital) in Shenzhen, China from March 2018 to May 2020 and who also had available baseline EMRs. The exclusion criteria were as follows: 1$)$ non-adult patients $(<18$ years); 2) patients with autoimmune, allergic, or infectious diseases that affect the EMR. This study was approved by the Ethics Committee of Fuwai Hospital of the Chinese Academy of Medical Sciences, Shenzhen, China.

\section{Data Collection and Definitions}

The sampling procedure was performed by reviewing the electronic medical records of the Fuwai Hospital Chinese Academy of Medical Sciences and screening the population based on the inclusion and exclusion criteria. Data on basic patient demographics, clinical findings, laboratory test results, presence of complications, and medications used were extracted from the records. The eosinophil and monocyte absolute values from the first fasting blood sample during hospitalization were obtained from the hospital laboratory. Each comprehensive decompensated HF diagnosis was made according to the standards established by the European Society of Cardiology clinical practice guidelines and based on a combination of the patient's medical history, clinical findings, cardiac color Doppler ultrasound, and N-terminal pro B-type natriuretic peptide (NTproBNP) value. ${ }^{10}$ The eosinophil count was determined using an automatic blood cell analyzer, and the baseline EMR was calculated according to the following formula: absolute eosinophil value $\left(\times 10^{9} / \mathrm{L}\right) /$ absolute monocyte value $\left(\times 10^{9} / \mathrm{L}\right){ }^{8}$

\section{Endpoints}

The primary endpoint was a composite outcome of $\mathrm{CV}$ death or rehospitalization due to HF; the secondary endpoints were $\mathrm{CV}$ death and rehospitalization due to $\mathrm{HF}$ (evaluated individually). The first primary endpoint event that occurred during the follow-up period was used in the analysis. If the same event occurred multiple times, only the first event was used. The patients (or their family members) reported whether the patient experienced $\mathrm{CV}$ death or rehospitalization due to HF within 1 year of hospital discharge. The clinical outcomes of interest were confirmed by trained personnel who carefully reviewed the corresponding medical records; these personnel were not aware of any baseline characteristics through telephone contact with the patient or their family members.

\section{Statistical Analysis}

All the patients were divided into three groups according to the EMR tertiles. If a continuous variable conformed to a normal distribution, it is expressed as the mean \pm standard deviation; otherwise, it is expressed as the median and interquartile range. Categorical variables are expressed as numbers and percentages. The chi-square or Fisher's exact tests were used to analyze the differences in the categorical variables between the groups. The ANOVA or Kruskal-Wallis $H$-tests were used to analyze the differences in the continuous variables between the groups. The Kaplan-Meier method was used to draw a time-to-event curve. The difference in the event incidences among the three EMR groups was determined using the Log rank test. We performed a Cox proportional hazard regression analysis to estimate the hazard ratios (HRs) and 95\% confidence intervals (CIs) for the clinical outcomes. The CochranArmitage test was used to determine the correlation between the EMR and clinical outcomes. We used the following four models to adjust for confounding factors: Model 1 was adjusted for white blood cell count, the total bilirubin, D-dimer, creatinine, potassium, sodium, HbAlc, and NTproBNP values, ejection fraction, and left ventricular end 
diastolic diameter; Model 2 was adjusted for the variables in model 1 plus sex, age, body mass index, systolic blood pressure, and heart rate; Model 3 was adjusted for the variables in model 2 plus the New York Heart Association classification, myocardial infarction (MI), atrial fibrillation, and the use of diuretics, $\beta$ blockers, digoxin, angiotensin-converting enzyme inhibitors/angiotensin receptor blockers/angiotensin receptor neprilysin inhibitors, spironolactone, and SGLT-2 inhibitors. Statistical significance was set at $\mathrm{P}<0.05$. The statistical analyses were performed using the "DescTools" and "survival" packages in $\mathrm{R}$ version 4.0.3 ( $\mathrm{R}$ foundation for Statistical Computing, Vienna, Austria).

\section{Results}

A patient selection flow chart is shown in Figure 1. In the present study, 2531 patients with HF were initially screened, of which 2264 patients were found to have decompensated HF. A total of 381 of these patients were excluded, as they did not have recorded eosinophil or monocyte absolute values. Finally, 1883 patients met the inclusion criteria. These patients were divided into three approximately equal groups according to the EMR tertile (T1, EMR $\leq 0.15$; T2, $0.15<\mathrm{EMR} \leq 0.32$; T3, EMR $>0.32$ ); T1 had 627 patients, T2 had 628 , and T3 had 628 . The baseline characteristics of the patients are shown in Table 1 . There were many significant differences between the baseline characteristics and the trends across the EMR tertiles. As the EMR increased across the tertiles, the proportions of men, age, blood pressure, sodium level, and use of angiotensin-converting enzyme inhibitors/angiotensin receptor blockers/angiotensin receptor neprilysin inhibitors increased. In contrast, higher EMRs across the tertiles also corresponded to lower heart rates, total bilirubin, D-dimer, and NT-proBNP levels, atrial fibrillation incidence, and diuretic and digoxin use.

\section{Primary Outcome}

The primary outcome survival curves of the three EMR groups are shown in Figure 2. There were significant differences in the composite outcome of $\mathrm{CV}$ death or HF rehospitalization event rates among the three groups (log-rank $\mathrm{P}=0.007$ ). A comparison between the groups is shown in Table 2. The results show that both the T1 group (HR: 1.50, 95\% CI: 1.16-1.94, $\mathrm{P}=0.002$ ) and the T2 group (HR: 1.34, 95\% CI: $1.03-1.74, \mathrm{P}=0.030$ ) had higher risks of $\mathrm{CV}$ death or HF rehospitalization compared to the $\mathrm{T} 3$ group. The T1 group (HR: $1.43,95 \% \mathrm{CI}: 1.09-1.87, \mathrm{P}=0.010$ ) and the T2 group (HR: 1.34, 95\% CI: 1.03-1.74, $\mathrm{P}=0.032$ ) also both show higher risks of $\mathrm{CV}$ death or HF rehospitalization than the T3 group in adjusted model 1 . In model 2 , the $\mathrm{T} 1$ group (HR: $1.43,95 \% \mathrm{CI}: 1.08-1.88, \mathrm{P}=0.011$ ) and the T2 group (HR: 1.36, 95\% CI: 1.04-1.78, $\mathrm{P}=0.023$ ) both show higher risks of $\mathrm{CV}$ death or $\mathrm{HF}$ rehospitalization than the $\mathrm{T} 3$ group. In model 3, both the T1 group (HR: 1.34, 95\% CI: $1.01-$ 1.77, $\mathrm{P}=0.040)$ and the T2 group (HR: 1.33 , 95\% CI: $1.02-$ $1.74, \mathrm{P}=0.035$ ) had higher risks of $\mathrm{CV}$ death or HF rehospitalization compared to the T3 group. The Cochran-Armitage

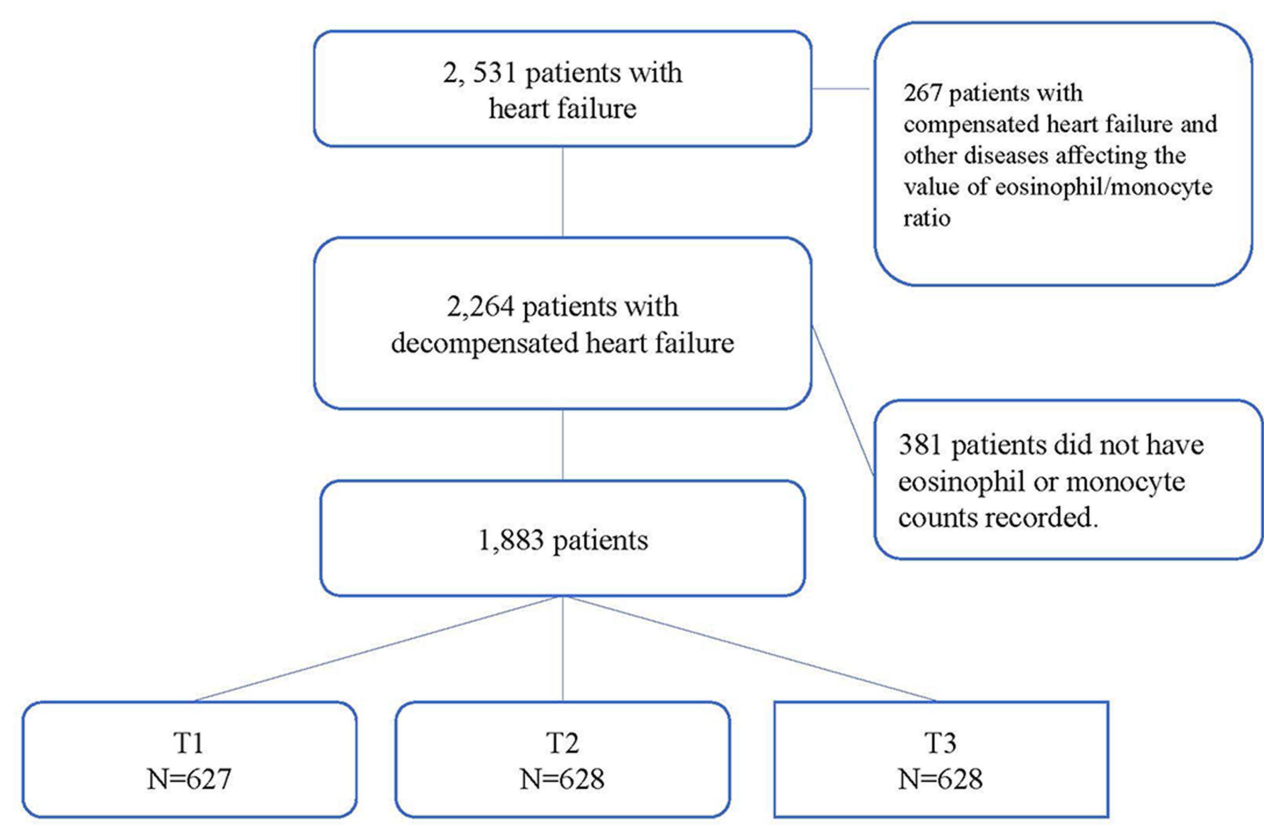

Figure I Flow chart of patient selection. 
Table I The Characteristic of Population

\begin{tabular}{|c|c|c|c|c|}
\hline & TI $(E M R \leq 0.15)$ & T2 $(0.15<E M R \leq 0.32)$ & T3 $(E M R>0.32)$ & $P$ value \\
\hline Number & 627 & 628 & 628 & \\
\hline Age (mean (SD)) & $59.93(14.51)$ & $59.96(14.91)$ & $61.93(13.86)$ & 0.019 \\
\hline Male (\%) & $308(49.1)$ & $344(54.8)$ & $377(60.0)$ & 0.001 \\
\hline BMI (mean (SD)) & $20.90(1.91)$ & $21.01(1.76)$ & $21.13(1.82)$ & 0.078 \\
\hline $\mathrm{SBP}($ mean $(\mathrm{SD}))$ & $121.35(20.53)$ & $124.26(20.00)$ & $125.53(21.68)$ & 0.001 \\
\hline Heart rate (mean $(S D))$ & $85.34(20.54)$ & 79.87 (19.44) & 78.19 (I7.7I) & $<0.001$ \\
\hline WBC (mean (SD)) & $8.78(3.55)$ & $7.29(2.55)$ & $6.67(2.03)$ & $<0.001$ \\
\hline TB (mean (SD)) & $20.05(16.07)$ & $16.02(11.45)$ & $13.50(9.34)$ & $<0.001$ \\
\hline D-dimer (mean (SD)) & $2.69(4.46)$ & $1.30(2.61)$ & $0.90(1.49)$ & $<0.001$ \\
\hline Creatinine (mean (SD)) & $101.20(58.42)$ & $99.36(61.77)$ & $104.17(68.62)$ & 0.394 \\
\hline Potassium (mean (SD)) & $4.25(0.36)$ & $4.18(0.35)$ & $4.25(0.38)$ & $<0.001$ \\
\hline Sodium (mean (SD)) & I39.49 (3.1I) & I $40.38(2.84)$ & $140.63(2.83)$ & $<0.001$ \\
\hline HbAlc (mean (SD)) & $5.87(1.02)$ & $5.92(1.13)$ & $5.97(1.18)$ & 0.262 \\
\hline \multicolumn{5}{|l|}{ NT-proBNP } \\
\hline (median (range)) & $4333.4 I(6422.14)$ & $2655.52(4835.77)$ & $2209.65(4392.10)$ & $<0.001$ \\
\hline $\mathrm{EF}($ mean $(\mathrm{SD}))$ & $42.84(12.28)$ & $43.67(11.65)$ & 43.33 (1 I.44) & $0.46 I$ \\
\hline LVEDD (mean (SD)) & $53.56(11.84)$ & $53.36(11.82)$ & $53.59(10.4 I)$ & 0.925 \\
\hline Cause of HF (\%) & & & & 0.002 \\
\hline DCM & $276(44.0)$ & $315(50.2)$ & $309(49.2)$ & \\
\hline ICM & $110(17.5)$ & $109(17.4)$ & $96(15.3)$ & \\
\hline VHD & $47(7.5)$ & $58(9.2)$ & $79(12.6)$ & \\
\hline HCM & $162(25.8)$ & $119(18.9)$ & $106(16.9)$ & \\
\hline NYHA classification (\%) & & & & $<0.001$ \\
\hline III & $36(5.7)$ & $86(13.7)$ & $108(17.2)$ & \\
\hline IV & $190(30.3)$ & $24 I(38.4)$ & $274(43.6)$ & \\
\hline Hypertension (\%) & $42(6.7)$ & $40(6.4)$ & $58(9.2)$ & 0.106 \\
\hline MI (\%) & $54(8.6)$ & $63(10.0)$ & $67(10.7)$ & 0.454 \\
\hline $\mathrm{AF}(\%)$ & $272(43.4)$ & $212(33.8)$ & $200(31.8)$ & $<0.001$ \\
\hline Diuretics (\%) & $489(78.0)$ & $405(64.5)$ & $371(59.1)$ & $<0.001$ \\
\hline B blocker (\%) & $375(59.8)$ & $394(62.7)$ & $383(61.0)$ & 0.563 \\
\hline Digoxin (\%) & $143(22.8)$ & $77(12.3)$ & $58(9.2)$ & $<0.001$ \\
\hline ACEI/ARB/ARNI (\%) & $243(38.8)$ & $318(50.6)$ & $329(52.4)$ & $<0.001$ \\
\hline Spironolactone (\%) & $260(4 \mid .5)$ & $228(36.3)$ & $233(37.1)$ & 0.129 \\
\hline
\end{tabular}

Abbreviations: BMI, body mass index; SBP, systolic blood pressure; WBC, white blood cell; TB, Total bilirubin; EF, ejection fraction; LVEDD, left ventricular end-diastolic diameter; HF, heart failure; NYHA, New York Heart Association; MI, myocardial infarction; AF, atrial fibrillation; ACEl, angiotensin converting enzyme inhibitors; ARB, angiotensin II receptor blockers; ARNI, angiotensin receptor neprilysin inhibitor; DCM, dilated cardiomyopathy; ICM, ischemic cardiomyopathy; VHD, valvular heart disease; HCM, hypertrophic cardiomyopathy. 


\section{Kaplan-Meier Curves}

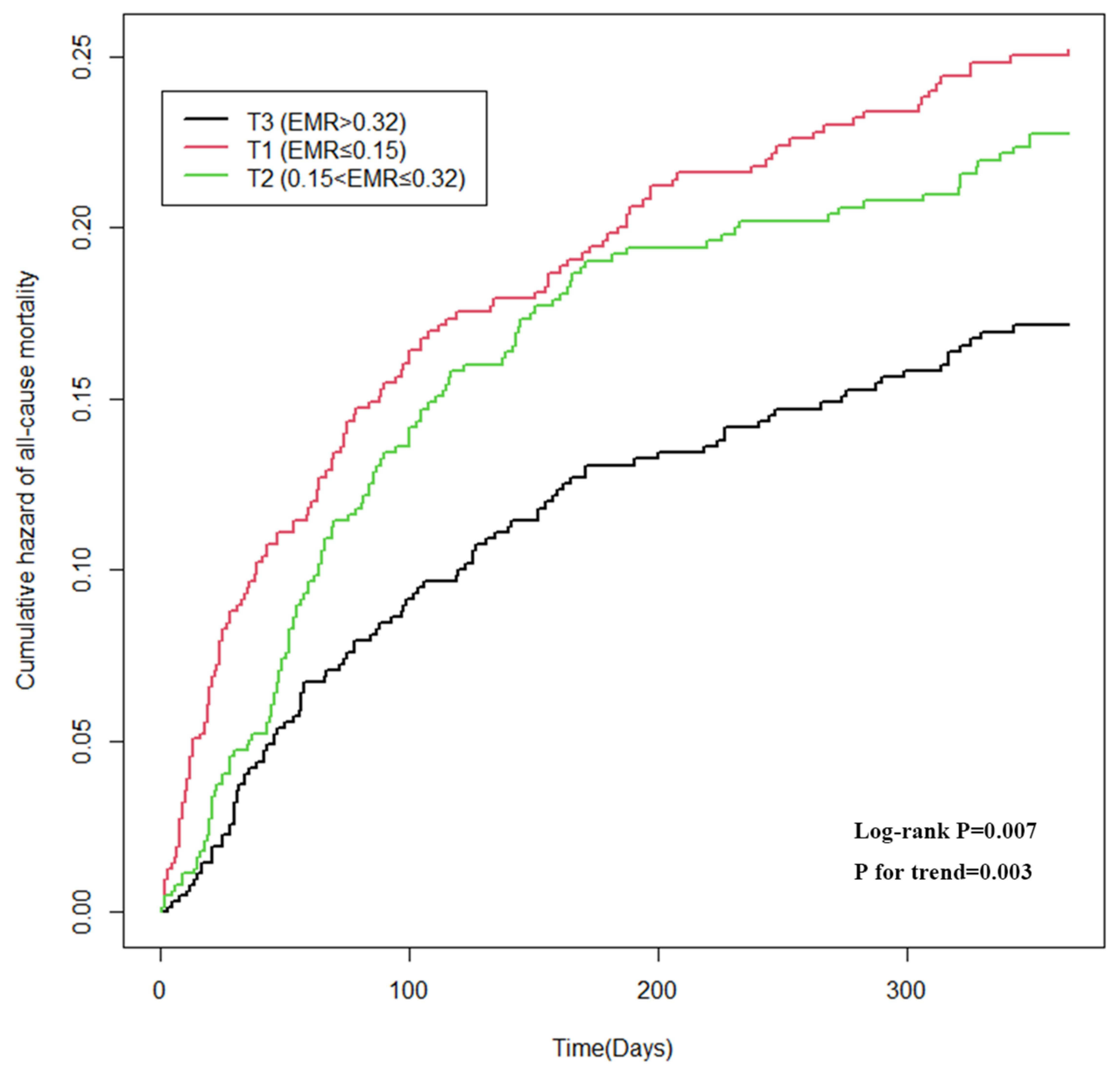

Figure 2 Kaplan-Meier survival curve for the primary endpoint event.

test for trends showed a positive correlation between the EMR and the primary outcome $(\mathrm{P}=0.003)$.

\section{Cardiovascular Death}

The CV death endpoint survival curve is shown in Figure 3. There were significant differences in the risk of $\mathrm{CV}$ death among the three groups (log-rank $\mathrm{P}<0.001$ ). A results comparison between the groups is presented in Table 2. Compared with the $\mathrm{T} 3$ group, the $\mathrm{T} 1$ group had a higher risk of CV death (HR: 4.24, 95\% CI: 1.86-9.67, $\mathrm{P}<0.001$ ), and the T2 group had a similar risk of $\mathrm{CV}$ death (HR, 1.29; 95\% CI: 0.48-3.46, $\mathrm{P}=0.615$ ). The CochranArmitage test for trend showed a positive correlation between the EMR and CV death $(\mathrm{P}<0.001)$.

\section{Heart Failure Rehospitalization}

The HF rehospitalization endpoint survival curve is shown in Figure 4. There were significant differences in the risk of $\mathrm{CV}$ death among the three groups (log-rank $\mathrm{P}=0.030$ ). A results comparison between the groups is presented in Table 2. Compared with the T3 group, the T1 group had a similar risk of CV death (HR: 1.25, 95\% CI: 0.95-1.64, $\mathrm{P}=0.110$ ), and the $\mathrm{T} 2$ group had a higher risk of $\mathrm{CV}$ death (HR, 1.44; 95\% CI, 1.10-1.88; $\mathrm{P}=0.008$ ). The CochranArmitage test for trend showed no correlation between the EMR and HF rehospitalization $(\mathrm{P}=0.134)$.

\section{Discussion}

The results of this study show that there is a correlation between the EMR and the composite risk of death or rehospitalization for patients with decompensated HF; ie, the higher the $\mathrm{HF}$ ratio, the lower the composite risk of $\mathrm{CV}$ death or HF rehospitalization.

Resident and recruited immune cells play a role in heart damage. ${ }^{11-13}$ Initially, resident and infiltrating immune cells activate inflammatory pathways and tissue 
Table 2 The Association Between Eosinophil/Monocyte Ratio and Clinical Outcomes in Patients with Decompensated Heart Failure

\begin{tabular}{|c|c|c|c|c|c|}
\hline & TI $(E M R \leq 0.15)$ & T2 $(0.15<E M R \leq 0.32)$ & T3 $(E M R>0.32)$ & Log Rank Test (P value) & $P$ for Trend \\
\hline Number & 627 & 628 & 628 & & \\
\hline \multicolumn{6}{|l|}{ Primary outcome } \\
\hline Events & 140 & 128 & 99 & \multirow{5}{*}{0.007} & \multirow{5}{*}{0.003} \\
\hline $\mathrm{HR}(95 \% \mathrm{Cl})$ & $1.50(1.16-1.94)$ & $1.34(1.03-1.74)$ & References & & \\
\hline Adjusted Model I & $1.43(1.09-1.87)$ & $1.34(1.03-1.74)$ & References & & \\
\hline Adjusted Model 2 & 1.43 (I.08-1.88) & $1.36(1.04-1.78)$ & References & & \\
\hline Adjusted Model 3 & $1.34(1.01-1.77)$ & $1.33(1.02-1.74)$ & References & & \\
\hline \multicolumn{6}{|l|}{ Cardiovascular dead } \\
\hline Events & 29 & 9 & 7 & & \\
\hline $\mathrm{HR}(95 \% \mathrm{Cl})$ & $4.24(1.86-9.67)$ & $1.29(0.48-3.46)$ & References & $<0.001$ & $<0.001$ \\
\hline \multicolumn{6}{|l|}{ HF hospitalization } \\
\hline Events & 114 & 123 & 94 & & \\
\hline $\mathrm{HR}(95 \% \mathrm{Cl})$ & $1.25(0.95-1.64)$ & I.44 (I.10-1.88) & References & 0.03 & 0.134 \\
\hline
\end{tabular}

Abbreviations: $\mathrm{HR}$, hazard ratio; $\mathrm{Cl}$, confidence interval; $\mathrm{EMR}$, eosinophil/monocyte ratio; HF, heart failure.

repair processes that determine the course of $\mathrm{HF}$ development. ${ }^{14}$ Immune cells not only regulate the function of cardiomyocytes, but they also regulate the progress of interstitial fibrosis, thereby affecting heart function. ${ }^{15}$ Patients with HF often have an accompanied increase in proinflammatory cytokine levels, which becomes more obvious as heart function deteriorates. ${ }^{16}$ Therefore, inflammatory mechanisms may be involved in the HF pathophysiological process. ${ }^{17}$ Indeed, increased levels of proinflammatory cytokines and other inflammatory markers may identify patients at risk of developing HF. ${ }^{5,18}$

The EMR is a new marker that can reflect the level of inflammation in the body. Deng et al conducted a retrospective study to evaluate the value of the EMR for predicting major adverse cardiovascular events in patients with MI, and their results indicated that there is a positive correlation. ${ }^{9}$ Another study indicated that the EMR can predict prognosis and functional outcomes in patients with ischemic stroke. ${ }^{78}$ Inflammatory factors are thought to be involved in the occurrence and progression of ischemic CV disease, and the level of inflammation is related to its prognosis. ${ }^{19}$ Clinical studies have shown that the use of inflammatory inhibitors can improve the prognosis of patients with ischemic CV disease. ${ }^{20-22}$ Although the EMR reflects the level of inflammation in the body, the predictive value of this ratio for the prognosis of patients with HF remains unclear. In the present study, a retrospective analysis method was used to explore this issue, and the results showed that the EMR and the prognosis of patients with chronic HF were positively correlated.
There are several possible explanations for this finding. Eosinophils may mediate myocardial tissue repair. Animal experiments have shown that eosinophil proliferation can promote the subsequent polarization of IL-4/STAT6 axismediated macrophages to the M2 type, thereby promoting cardiac structure and function recovery after an MI. ${ }^{23}$ Liu et al reported that blood and cardiac eosinophil counts increased after MIs in both humans and mice; these increases were primarily in the infarcted area, and the authors established the cardioprotective effect of eosinophils in the heart post-MI. ${ }^{24}$ An increase in peripheral monocytes can also occur 2-3 days after an acute infarction, indicating monocyte and macrophage infiltration into the necrotic myocardium. ${ }^{25}$ Higher peak monocyte levels are associated with larger left ventricular end-diastolic volumes, lower left ventricular ejection fractions, and worse clinical outcomes. ${ }^{26-28}$ Additionally, a peak monocyte count $>900$ can independently predict HF, left ventricular aneurysm formation, and cardiac events. ${ }^{29}$ Therefore, eosinophils and monocytes have opposite effects on HF; ie, higher eosinophil numbers and lower monocyte numbers both correlate with better patient prognoses.

The EMR, which directly reflects the prognosis of patients, is easy to obtain in clinical practice. In future research, the EMR can be incorporated into existing HF prognostic prediction models to improve their predictive performance. Additionally, the results of this study confirm that not all inflammatory indicators are harmful for patients with HF. For example, an increase in eosinophils during the early stages of HF may have a protective effect 


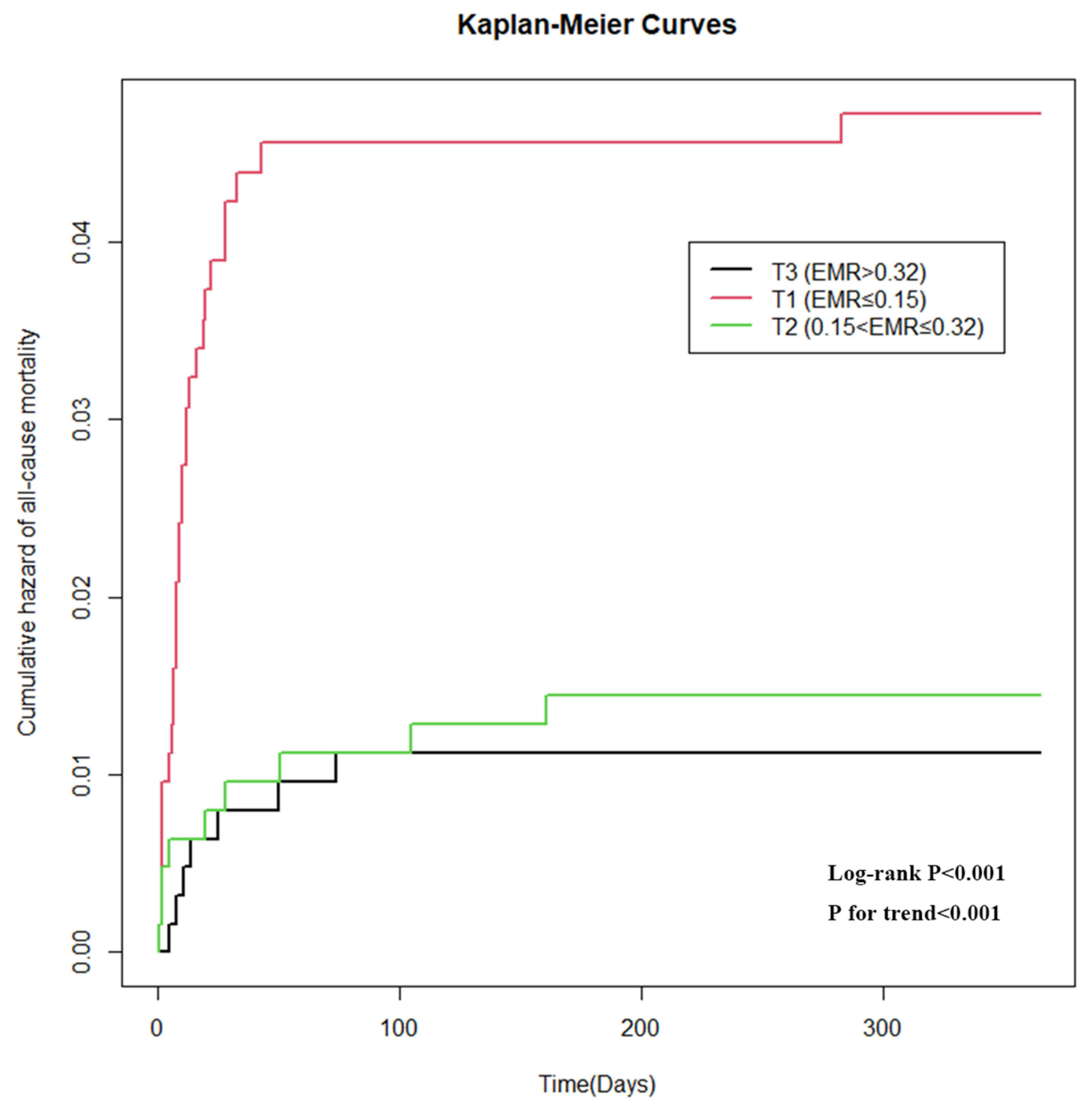

Figure 3 Kaplan-Meier survival curve for cardiovascular death.

on cardiomyocytes. However, clinical trials have failed to confirm that inflammation inhibitors can improve the prognosis of patients with $\mathrm{HF}^{30-32}$ suggesting that there are multiple inflammatory pathways involved in its occurrence and progression. Further research is needed to explore the pathophysiological mechanisms underlying the influence of eosinophils on the structure and function of the heart.

\section{Limitations}

This study has several limitations. First, the follow-up time was too short; a longer follow-up period is needed to clarify the influence of the EMR on long-term prognoses. Second, causality could not be determined because this was a single-center retrospective study. Third, this study was confined to a population of Chinese patients. Further research is needed to verify whether our findings are valid for other populations. Fourth, we obtained the first EMR for each patient at admission. However, the EMR was a time-dependent covariate. Although the proportional hazards assumption from the Cox regression model held, and we used the Cox regression model to estimate the effect of the EMR on survival, further studies using a Cox model with time-dependent covariates and data that has been organized in a counting process style are warranted. ${ }^{33}$ Fifth, there are different pathogenesis mechanisms of preserved and reduced HF development. However, the patients in our study were not sorted according to the ejection fraction. Therefore, further studies are warranted to evaluate whether the type of HF (HF with preserved, mid-range, or reduced ejection fraction) influences the effect of the EMR on prognosis.

\section{Conclusions}

The results of this study show that the EMR is positively correlated with the prognosis of patients with decompensated HF; ie, the lower the ratio, the worse the prognosis. 
Kaplan-Meier Curves

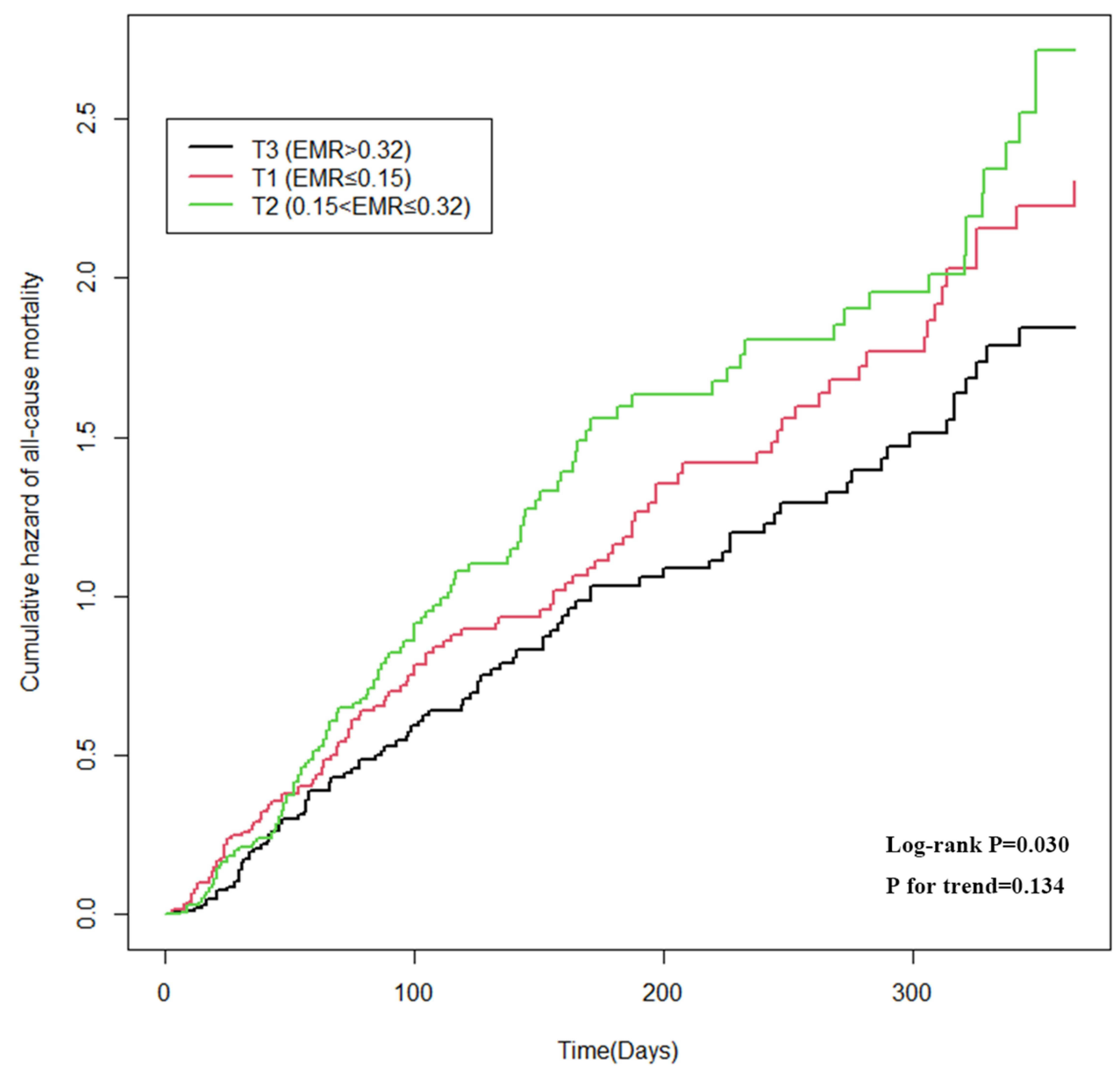

Figure 4 Kaplan-Meier survival curve for rehospitalization due to heart failure.

Further studies are needed to evaluate the impact of dynamic monitoring of the EMR on the prognosis of patients with HF.

\section{Abbreviations}

ANOVA, analysis of variance; $\mathrm{CI}$, confidence interval; CV, cardiovascular; EMR, eosinophil/monocyte ratio; HBA1C, hemoglobin A1C; HF, heart failure; HR, hazard ratio; IL4/STAT6, interleukin-4/signal transducer and activator of transcription 6; MI, myocardial infarct; NT-proBNP, N-terminal pro B-type natriuretic peptide; SGLT-2 inhibitors, sodium-glucose co-transporter- 2 inhibitors; TNF- $\alpha$, tumor necrosis factor-alpha.

\section{Data Sharing Statement}

All the data reported in this study are available upon request by contacting the corresponding author.

\section{Ethical Statement}

This study was approved by the Ethics Committee of Fuwai Hospital of the Chinese Academy of Medical Sciences, Shenzhen and was performed in accordance with the Declaration of Helsinki. Informed consent was waived due to this study's retrospective nature and the anonymized processing of patient data.

\section{Author Contributions}

All authors made a significant contribution to the work reported, whether that is in the conception, study design, execution, acquisition of data, analysis, and interpretation, or in all these areas; took part in drafting, revising or critically reviewing the article; gave final approval of the version to be published; have agreed on the journal to which the article has been submitted; and agree to be accountable for all aspects of the work. 


\section{Funding}

This study was supported by Shenzhen Science and Technology Research Funding (No. 20170502165510880; JCYJ20180302173854598) and the Fund of "Sanming," Project of Medicine in Shenzhen (No. SZSM201603072).

\section{Disclosure}

Xiehui Chen and Weichao Huang are co-first authors for this study. The authors report no conflicts of interest in this work.

\section{References}

1. Zhang Z, Cao L, Chen R, et al. Electronic healthcare records and external outcome data for hospitalized patients with heart failure. $S c i$ Data. 2021;8:46. doi:10.1038/s41597-021-00835-9

2. Pons F, Lupon J, Urrutia A, et al. Mortality and cause of death in patients with heart failure: findings at a specialist multidisciplinary heart failure unit. Rev Esp Cardiol. 2010;63:303-314. doi:10.1016/ S0300-8932(10)70089-0

3. Azad N, Lemay G. Management of chronic heart failure in the older population. J Geriatr Cardiol. 2014;11:329-337.

4. Dunlay SM, Weston SA, Redfield MM, Killian JM, Roger VL. Tumor necrosis factor-alpha and mortality in heart failure: a community study. Circulation. 2008;118:625-631. doi:10.1161/ CIRCULATIONAHA.107.759191

5. Hage C, Michaelsson E, Linde C, et al. Inflammatory biomarkers predict heart failure severity and prognosis in patients with heart failure with preserved ejection fraction: a holistic proteomic approach. Circ Cardiovasc Genet. 2017;10:e01633. doi:10.1161/ CIRCGENETICS.116.001633

6. Seguela PE, Iriart X, Acar P, Montaudon M, Roudaut R, Thambo JB. Eosinophilic cardiac disease: molecular, clinical and imaging aspects. Arch Cardiovasc Dis. 2015;108:258-268. doi:10.1016/j.acvd.2015.01.006

7. Yu S, Luo Y, Zhang $\mathrm{T}$, et al. Eosinophil-to-monocyte ratio is a potential biomarker in the prediction of functional outcome among patients with acute ischemic stroke. BMC Neurosci. 2021;22:8. doi:10.1186/s12868-021-00610-x

8. Chen Y, Ren J, Yang N, et al. Eosinophil-to-monocyte ratio is a potential predictor of prognosis in acute ischemic stroke patients after intravenous thrombolysis. Clin Interv Aging. 2021;16:853-862. doi:10.2147/CIA.S309923

9. Deng X, Wang X, Shen L, et al. Association of eosinophil-tomonocyte ratio with 1-month and long-term all-cause mortality in patients with ST-elevation myocardial infarction undergoing primary percutaneous coronary intervention. $J$ Thorac Dis. 2018;10:54 49-5458. doi: 10.21037/jtd.2018.09.27

10. Ponikowski P, Voors AA, Anker SD, et al. 2016 ESC Guidelines for the diagnosis and treatment of acute and chronic heart failure: the Task Force for the diagnosis and treatment of acute and chronic heart failure of the European Society of Cardiology (ESC). Developed with the special contribution of the Heart Failure Association (HFA) of the ESC. Eur J Heart Fail. 2016;18:891-975.

11. Liu T, Song D, Dong J, et al. Current understanding of the pathophysiology of myocardial fibrosis and its quantitative assessment in heart failure. Front Physiol. 2017;8:238. doi:10.3389/fphys.2017. 00238

12. Bracamonte-Baran W, Cihakova D. Cardiac autoimmunity: myocarditis. Adv Exp Med Biol. 2017;1003:187-221.

13. Iacobazzi D, Suleiman MS, Ghorbel M, George SJ, Caputo M, Tulloh RM. Cellular and molecular basis of RV hypertrophy in congenital heart disease. Heart. 2016;102:12-17. doi:10.1136/ heartjnl-2015-308348
14. Mouton AJ, Rivera OJ, Lindsey ML. Myocardial infarction remodeling that progresses to heart failure: a signaling misunderstanding. $\mathrm{Am}$ J Physiol Heart Circ Physiol. 2018;315:H71-H79. doi:10.1152/ ajpheart.00131.2018

15. Strassheim D, Dempsey EC, Gerasimovskaya E, Stenmark K, Karoor V. Role of inflammatory cell subtypes in heart failure. J Immunol Res. 2019;2019:2164017. doi:10.1155/2019/2164017

16. Torre-Amione G, Kapadia S, Benedict C, Oral H, Young JB, Mann DL. Proinflammatory cytokine levels in patients with depressed left ventricular ejection fraction: a report from the Studies of Left Ventricular Dysfunction (SOLVD). J Am Coll Cardiol. 1996;27:1201-1206. doi:10.1016/0735-1097(95)00589-7

17. Aukrust P, Gullestad L, Ueland T, Damas JK, Yndestad A. Inflammatory and anti-inflammatory cytokines in chronic heart failure: potential therapeutic implications. Ann Med. 2005;37:74-85. doi:10.1080/07853890510007232

18. Vasan RS, Sullivan LM, Roubenoff R, et al. Inflammatory markers and risk of heart failure in elderly subjects without prior myocardial infarction: the Framingham Heart Study. Circulation. 2003; 107:1486-1491. doi:10.1161/01.CIR.0000057810.48709.F6

19. St-Pierre AC, Cantin B, Bergeron J, et al. Inflammatory markers and long-term risk of ischemic heart disease in men A 13-year follow-up of the Quebec Cardiovascular Study. Atherosclerosis. 2005; 182:315-321. doi:10.1016/j.atherosclerosis.2005.02.009

20. Kosmas CE, Silverio D, Sourlas A, Montan PD, Guzman E, Garcia MJ. Anti-inflammatory therapy for cardiovascular disease. Ann Transl Med. 2019;7:147. doi:10.21037/atm.2019.02.34

21. Ridker PM, Everett BM, Thuren T, et al. Antiinflammatory therapy with canakinumab for atherosclerotic disease. $N$ Engl $J$ Med. 2017;377:1119-1131. doi:10.1056/NEJMoa1707914

22. Nidorf SM, Fiolet ATL, Mosterd A, et al. Colchicine in patients with chronic coronary disease. $N$ Engl J Med. 2020;383(19):1838-1847. doi:10.1056/NEJMoa2021372

23. Peng H, Sarwar Z, Yang XP, et al. Profibrotic role for interleukin-4 in cardiac remodeling and dysfunction. Hypertension. 2015;66:58 2-589. doi:10.1161/HYPERTENSIONAHA.115.05627

24. Liu J, Yang C, Liu T, et al. Eosinophils improve cardiac function after myocardial infarction. Nat Commun. 2020;11:6396. doi:10.1038/ s41467-020-19297-5

25. Meisel SR, Pauzner H, Shechter M, Zeidan Z, David D. Peripheral monocytosis following acute myocardial infarction: incidence and its possible role as a bedside marker of the extent of cardiac injury. Cardiology. 1998;90:52-57. doi:10.1159/000006817

26. Wang KT, Liu YY, Sung KT, et al. Circulating monocyte count as a surrogate marker for ventricular-arterial remodeling and incident heart failure with preserved ejection fraction. Diagnostics. 2020;10 (5):287. doi:10.3390/diagnostics 10050287

27. Charach G, Rogowski O, Karniel E, Charach L, Grosskopf I, Novikov I. Monocytes may be favorable biomarker and predictor of long-term outcome in patients with chronic heart failure: a cohort study. Medicine. 2019;98:e17108. doi:10.1097/MD.000000000001 7108

28. Mongirdiene A, Laukaitiene J, Skipskis V, Kursvietiene L, Liobikas J. Platelet activity and its correlation with inflammation and cell count readings in chronic heart failure patients with reduced ejection fraction. Medicina. 2021;57:176.

29. Maekawa Y, Anzai T, Yoshikawa T, et al. Prognostic significance of peripheral monocytosis after reperfused acute myocardial infarction: a possible role for left ventricular remodeling. $\mathrm{J} \mathrm{Am} \mathrm{Coll} \mathrm{Cardiol.}$ 2002;39:241-246. doi:10.1016/S0735-1097(01)01721-1

30. Chung ES, Packer M, Lo KH, Fasanmade AA, Willerson JT; Anti TNFTACHFI. Randomized, double-blind, placebo-controlled, pilot trial of infliximab, a chimeric monoclonal antibody to tumor necrosis factor-alpha, in patients with moderate-to-severe heart failure: results of the anti-TNF Therapy Against Congestive Heart Failure (ATTACH) trial. Circulation. 2003;107:3133-3140. 
31. Bozkurt B, Torre-Amione G, Warren MS, et al. Results of targeted anti-tumor necrosis factor therapy with etanercept (ENBREL) in patients with advanced heart failure. Circulation. 2001;103:10 44-1047. doi:10.1161/01.CIR.103.8.1044

32. Mann DL, McMurray JJ, Packer M, et al. Targeted anticytokine therapy in patients with chronic heart failure: results of the Randomized Etanercept Worldwide Evaluation (RENEWAL). Circulation. 2004; 109:1594-1602. doi:10.1161/01.CIR.0000124490.27666.B2
33. Zhang Z, Reinikainen J, Adeleke KA, Pieterse ME, GroothuisOudshoorn CGM. Time-varying covariates and coefficients in Cox regression models. Ann Transl Med. 2018;6:121. doi:10.21037/ atm.2018.02.12

\section{Publish your work in this journal}

The Journal of Inflammation Research is an international, peerreviewed open-access journal that welcomes laboratory and clinical findings on the molecular basis, cell biology and pharmacology of inflammation including original research, reviews, symposium reports, hypothesis formation and commentaries on: acute/chronic inflammation; mediators of inflammation; cellular processes; molecular mechanisms; pharmacology and novel anti-inflammatory drugs; clinical conditions involving inflammation. The manuscript management system is completely online and includes a very quick and fair peerreview system. Visit http://www.dovepress.com/testimonials.php to read real quotes from published authors. 